\title{
Toward a new computer-based and easy-to-use tool for the objective measurement of motivational states in humans: a pilot study
}

Bruno Aouizerate ${ }^{1,2,3,6^{*}}$, Camille Gouzien ${ }^{1,2,3,6}$, Olivier Doumy ${ }^{1,2,3,6}$, Pierre Philip ${ }^{3,4}$, Catherine Semal ${ }^{3,5}$, Laurent Demany ${ }^{3,5}$, Pier Vincenzo Piazza ${ }^{2,3}$ and Daniela Cota $2,3,6$

\begin{abstract}
Background: The experimental methods currently used for assessing motivational processes in humans have two major limitations. Some of them rely on global subjective assessments while others evaluate these processes using food-related tasks often coupled with functional neuroimaging techniques that have however limited availability and important associated costs. Here we propose a novel experimental computer-generated and easy-to-use tool primarily based on the presentation of food images and designed to provide a quantitative and objective measurement of motivational states in humans.
\end{abstract}

Methods: Two tasks evaluating respectively visual and time discrimination capacities were developed and tested on a sample of 30 healthy subjects. The subjects were asked to compare a food stimulus (food picture in color) and its devalued counterpart (same image in grayscale), at each trial, assessing either the size (task A) or the duration of presentation (task B). Geometric figures presented in color or grayscale were used as controls. The subjects were invited to perform tasks A and B during three separate experimental sessions, one under fasting and two under satiety.

Results: Relative to their devalued counterparts, the food images were judged significantly greater in size and shorter in time of presentation in fasting than in satiety. In fasting, the size and the time of presentation for the food images were respectively estimated significantly greater and shorter than for the control images when compared to their respective devalued counterparts. Conversely, there was no overall change in the perception of size or duration of presentation for the control images between fasting and satiety conditions.

Conclusions: Our findings support that hunger specifically affects the perception of visual food stimuli, and suggest that this novel computer-based test is potentially useful for the study of motivational states in human diseases that are characterized by serious disturbances in reward processing.

Keywords: Motivation, Computer-based tasks, Food images, Psychophysics

\section{Background}

Given the increasing prevalence of highly disabling pathologies, such as major depression, addiction and obesity, in which reward function is especially disrupted (Eaton et al. 2007; Merikangas and McClair 2012; Wang et al. 2011), there is a significant need for an easy-to-use

\footnotetext{
* Correspondence: bruno.aouizerate@u-bordeaux2.fr

${ }^{1}$ Regional medical center for the management and treatment of anxiety and depressive disorders, Centre Hospitalier Charles Perrens, F-33076 Bordeaux, France

${ }^{2}$ INSERM, Neurocentre Magendie, Physiopathologie de la Plasticité Neuronale, U862, F-33000 Bordeaux, France

Full list of author information is available at the end of the article
}

instrumental method designed to provide an accurate measurement of motivational states in humans.

Motivation (i.e. wanting), as one of the two components of reward beside the hedonic experience and sensory pleasure (i.e. liking), relies on the brain process involved in the attribution of incentive salience and that generates the desire to consume appetitive food (Berridge 1996; Berridge 2003; Finlayson et al. 2007; Finlayson and Dalton 2012; Cota et al. 2006; Piazza et al. 2007). The motivation to obtain and eat food is modulated by the sensations of hunger, as reflective of the physiological need to introduce calories (Berridge 1996; Berridge 2003; Finlayson et al. 
2007; Finlayson and Dalton 2012; Cota et al. 2006; Piazza et al. 2007).

Several methodological approaches have been used to study motivational processes. Some clinical investigations have tried to explore time experience (e.g. how time passes slowly or quickly) or judgement (e.g. how the duration of a given timespan is estimated or produced), as indicative of the degree of motivation, which is expected to be low when time is perceived long (Mezey and Cohen 1961; Wyrick and Wyrick 1977; Bschor et al. 2004). However, these studies have some limitations due to the frequent use of subjective assessment methods. Some authors have instead examined the processing of the motivational value of food visual cues (Stoeckel et al. 2008; Stoeckel et al. 2009). Such experimental paradigms coupled with functional neuroimaging allow objectively identifying the anatomofunctional correlates of the internal affective state. However, an important drawback is the limited availability and costly procedures associated with functional neuroimaging. There are other computer-based tests that primarily refer to: i) food reinforcement tasks for the study of motivated responses and effort toward food (Epstein et al. 2003; Giesen et al. 2010); ii) food tasting tasks for the evaluation of the hedonic experience and pleasantness elicited by food intake (Born et al. 2011; Cooke et al. 2011); and, iii) visual probe tasks with food images for the exploration of cognitive aspects and especially the attentional capture according to the motivational characteristics of food pictures (Di Pellegrino et al. 2011; Nijs et al. 2010). Thus, to date little attention has been paid to methods assessing motivational states in relation with the perception. While the manipulation of the emotional valence of words has recently been documented to create substantial changes in the size or time perception (Ode et al. 2012), the perceptual processing of motivationally significant stimuli such as food, which could putatively be linked to hunger levels, has not been investigated.

Therefore, our pilot study had as objective the development and use of a new experimental computer-generated and easy-to-use test based on the presentation of visual food cues for the objective and quantitative measurement of motivational states in humans. We explored the influence of both incentive salience and physiological hunger on the visual and time perception. To this purpose, we recruited normal-weight, healthy subjects to perform two behavioral tasks, named task A and task B. These tasks respectively challenged visual and time discrimination capacities between two stimuli, a food image in color ("F") and its devalued counterpart in grayscale ("D") under either fasting or satiety conditions. Geometric figures in color ("C") and graycale ("D") were used as controls. We hypothesized that fasting specifically causes changes in the perception of either the size or the presentation duration of the food images. Measurements of perceptual changes were based on the assessment of either the point of subjective equality (PSE) (i.e. the ratio "F"/"D" or "C"/ "D" for which the stimulus "F" or "C" was judged equal to "D" in terms of size or presentation time) or the percentage of subjective discrimination (PSD) (i.e. the percentage of responses where the stimulus " $\mathrm{F}$ " or "C" was judged greater than " $\mathrm{D}$ " during the trials where the stimulus " $\mathrm{F}$ " or "C" was physically equal to "D", in terms of size or duration of presentation). Our study demonstrates that the subjects perceived food, but not control, images bigger in size and shorter in duration of presentation in fasting as compared to satiety. These data thus suggest that this novel computer-based test easily allows assessing quantitatively and objectively motivational states in humans, representing a potentially useful tool for the study of behavioral responses in subjects suffering from pathologies in which motivational states are altered.

\section{Results}

Hunger levels of the study population and appetitive properties of food images

Visual analogue scales (VAS) were used to assess both hunger levels and attractiveness of the food images shown to the subjects recruited for the study. As expected, assessment of hunger levels revealed profound differences across fasting and satiety conditions [condition effect, $F(2,58)=143.07, p<0.0001]$. Hunger scores were significantly higher during the fasting session than during the satiety sessions $(\mathrm{p}<0.0001)$. Ratings of appetitive properties of food pictures on VAS showed a mean score of $6.39( \pm$ sem $=0.45)$. Additionally, there was no significant difference in the appetitive value of the stimuli " $F$ " when assessed at the end of the experimental session in either fasting or satiety [condition effect, $F(1,28)=0.04, \mathrm{p}>0.85$ ] Therefore, the appetitive properties of the stimuli " $\mathrm{F}$ " were not estimated greater in fasting than in satiety.

\section{Visual and time perceptions changes in response to hunger levels}

Given the absence of significant difference in either the PSE or PSD for both images "F" and "C" between the two experimental sessions under satiety, the PSE and PSD calculated for either the stimuli " $\mathrm{F}$ " or " $\mathrm{C}$ " in the two satiety sessions were combined.

For task A, assessments of the PSE revealed negative values on the logarithmic scale for both types of stimuli "F" and "C" in either fasting or satiety (Figure 1A). This means that the subjects considered the images " $\mathrm{F}$ " or " $C$ " equal in size to their respective devalued counterparts " $D$ " while the images " $F$ " or " $C$ " were in reality smaller in size than " $\mathrm{D}$ ". Therefore, the subjects overestimated the size of both stimuli " $F$ " and " $C$ " relative to their respective devalued counterparts " $\mathrm{D}$ " under either fasting or satiety. However, there was a tendency toward 


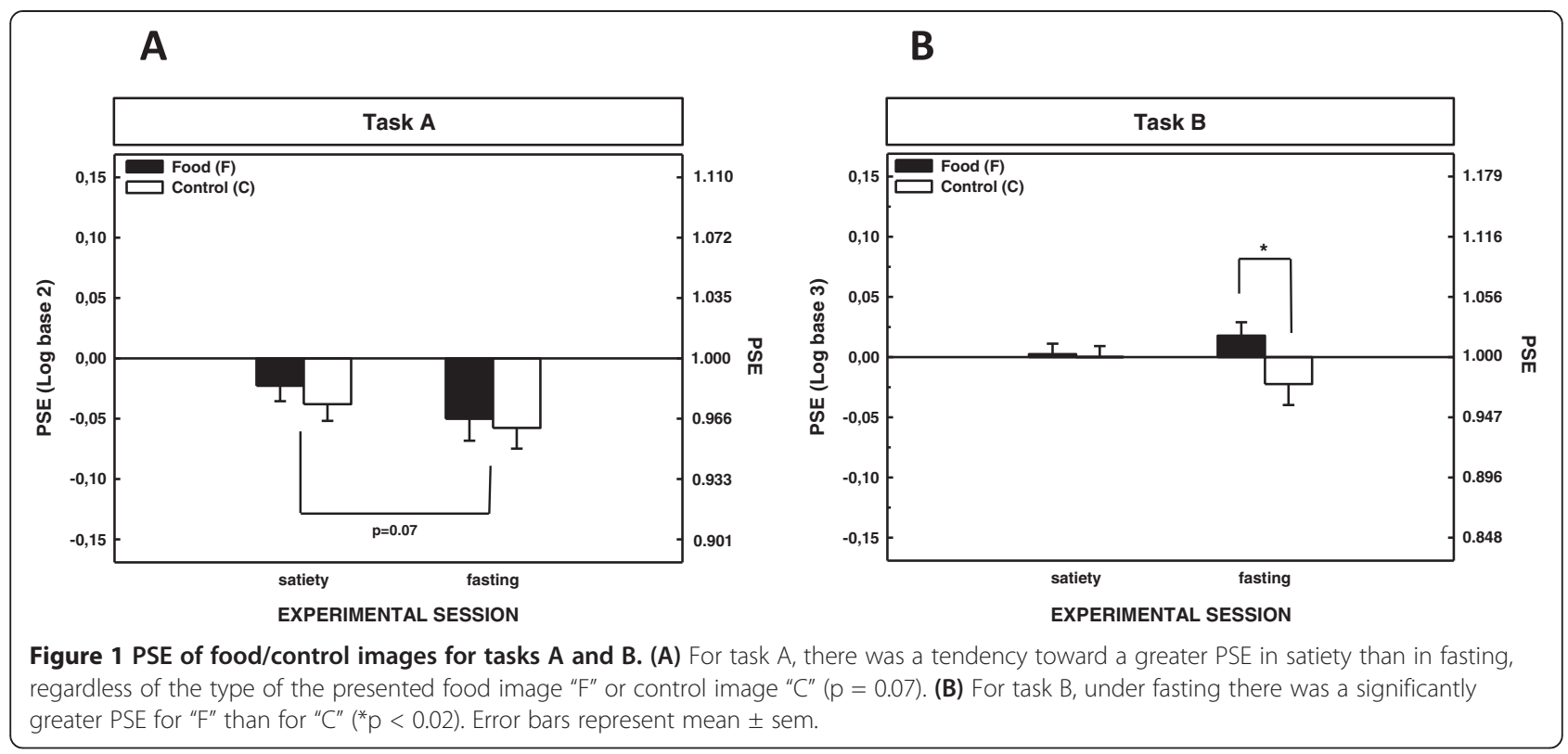

a difference in the PSE between fasting and satiety conditions, regardless of the type of the viewed stimuli "F" or "C" [condition effect, $F(1,23)=3.65, \mathrm{p}=0.07$ ]. The PSE of both images " $\mathrm{F}$ " and " $\mathrm{C}$ " tended to have more negative values on the logarithmic scale under fasting as compared to satiety (Figure 1A). Therefore, the overestimation seemed more marked under fasting than in satiety for both types of stimuli " $\mathrm{F}$ " and " $\mathrm{C}$ " when compared to their respective devalued counterparts " $\mathrm{D}$ ". In fasting, the PSE calculated for either the stimuli " $\mathrm{F}$ " or "C" was also found to be inversely correlated with the hunger levels measured by VAS [stimuli " $F$ ": $r=-0.43, p<0.04$; stimuli " $C$ ": $r=-0,45, p<0.03$ ]. In contrast, there was no significant relationship between the PSE of the stimuli " $\mathrm{F}$ " in fasting and their appetitive value as assessed by VAS at the end of the last experimental session $[r=-0.09, p>$ 0.68]. Analysis of the PSD revealed differences between the stimuli " $\mathrm{F}$ " and "C" across the two experimental conditions under fasting and satiety [stimulus $\mathrm{x}$ condition interaction, $\mathrm{F}(1,23)=4.50, \mathrm{p}<0.04$ ]. The PSD was significantly greater under fasting than in satiety for the images " $F$ " ( $\mathrm{p}<0.02)$, whereas there was no difference for the images " $\mathrm{C}$ " $(\mathrm{p}>0.78)$. Moreover, in fasting the PSD was significantly greater for the images " $\mathrm{F}$ " than for the images " $C$ " $(\mathrm{p}<0.03)$ (Figure 2A). Therefore, relative to their respective devalued counterparts, the stimuli " $F$ " under fasting were perceived greater in size than either the same type of stimuli in satiety or the stimuli " $\mathrm{C}$ " in fasting. However, the PSD calculated for the stimuli "F" was not significantly correlated with either the hunger levels in fasting $[r=0.10, p>0.68]$ or the appetitive value of the images measured by VAS $[r=0.14, p>0.53]$.
For task B, measurements of the PSE for the stimuli "F" and "C" varied differently across fasting and satiety conditions [stimulus $\mathrm{x}$ condition interaction, $\mathrm{F}(1,26)=$ 4.34, $\mathrm{p}<0.05$ ]. The PSE for the images "F" had a positive value on the logarithmic scale in fasting (Figure 1B). In other words, the subjects considered the stimuli " $F$ " equal in time of presentation to their devalued counterparts " $\mathrm{D}$ " while the stimuli " $\mathrm{F}$ " were in reality greater in time of presentation than " $D$ ". Therefore, in fasting, the subjects underestimated the duration of presentation of the stimuli " $F$ " when compared to their devalued counterparts " $D$ ". Opposite results were observed for the stimuli " $C$ ". The PSE for the images "C" showed a negative value on the logarithmic scale in fasting (Figure 1B). This means that the subjects perceived the stimuli "C" equal in time of presentation to their devalued counterparts " $D$ " while the stimuli " $C$ " were in reality smaller in time of presentation than " $\mathrm{D}$ ". In other words, in the fasting condition, the subjects overestimated the duration of presentation of the stimuli "C" when compared to their devalued counterparts "D". The PSE for the images "F" was significantly greater than that of the images " $\mathrm{C}$ " in fasting $(\mathrm{p}<0.02)$ (Figure $1 \mathrm{~B})$. Therefore, relative to their respective devalued counterparts " $\mathrm{D}$ ", the duration of presentation of the stimuli " $\mathrm{F}$ " was estimated significantly smaller than that of the stimuli " $\mathrm{C}$ " under fasting. Concordant data were obtained for the PSD [stimulus $\mathrm{x}$ condition interaction, $F(1,26)=7.59, p<0.01$ ] (Figure 2B). The PSD for the images "F" was significantly smaller than that of the images " $\mathrm{C}$ " in fasting $(\mathrm{p}<0.01)$. Additionally, there was a significantly smaller PSD under fasting than in satiety for the "F" images $(\mathrm{p}<0.01)$, whereas no difference 


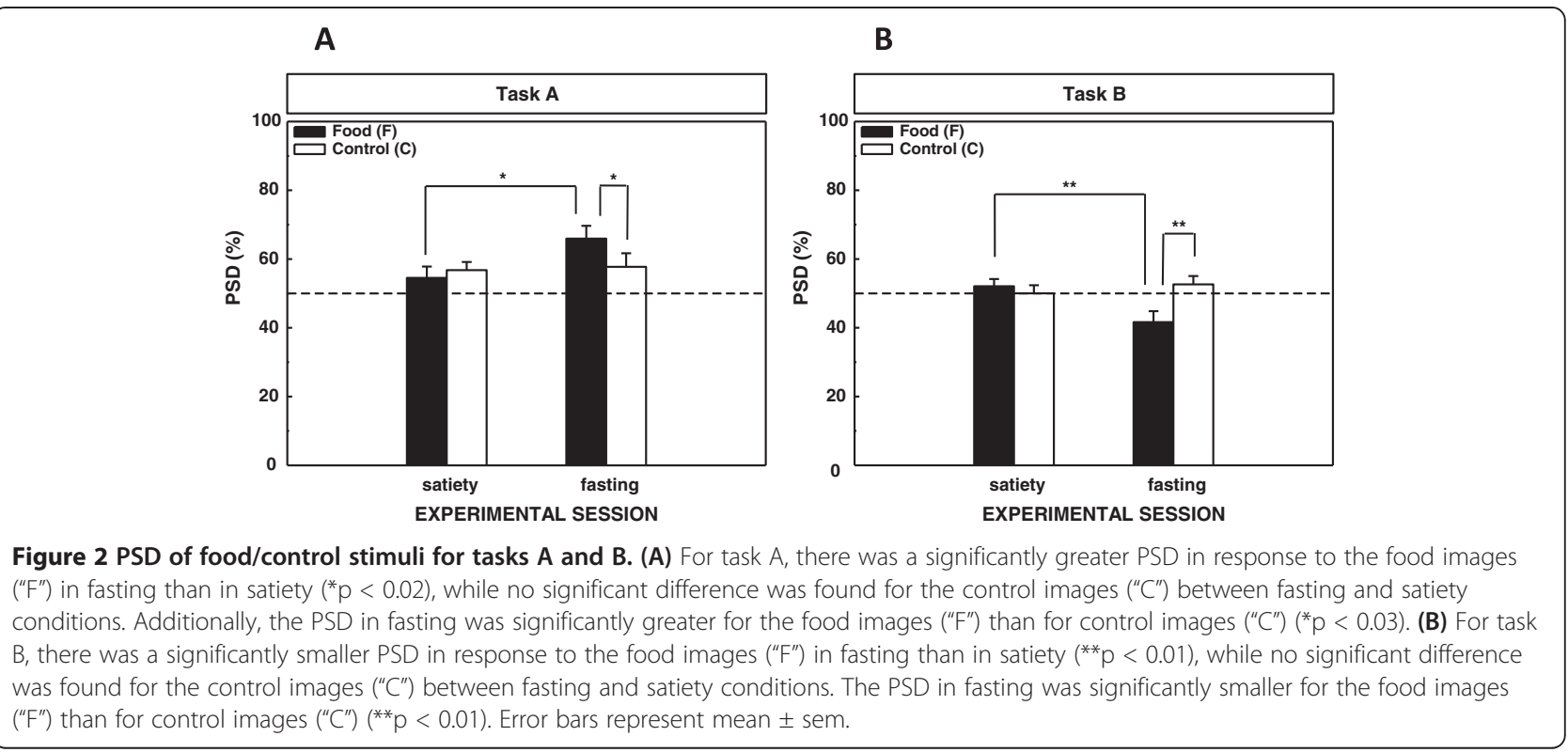

was observed for the " $\mathrm{C}$ " images $(\mathrm{p}>0.72)$ (Figure 2B). Thus, relative to their respective devalued counterparts, the stimuli " $F$ " under fasting were estimated shorter in duration of presentation than either "C" under fasting or "F" under satiety. However, the PSE or the PSD of the stimuli " $F$ " did not correlate either with hunger levels in fasting [PSE: $r=-0.21, p>0.29$; PSD: $r=-0.08, p>0.69$ ] or with the appetitive properties of the images measured by VAS [PSE: $\mathrm{r}=-0.04, \mathrm{p}>0.85$; PSD: $\mathrm{r}=0.01, \mathrm{p}>0.97$ ].

\section{Discussion}

To our knowledge, this is the first study using psychophysical methods for the development of a test based on perception for the objective and quantitative assessment of motivational states in humans.

In the studied subjects, hunger levels recorded in fasting were substantially higher than those in satiety. This was paralleled by changes in the estimated size of the selected food images, which was judged greater in fasting than in satiety, when compared to their devalued counterparts. Interestingly, these changes in the perception of size were inversely related with hunger levels in fasting. Thus, more the subjects were hungry, more the size of food images was overestimated in fasting. Concomitantly, relative to their devalued counterparts, the food images was considered shorter in duration of presentation in fasting than in satiety. Conversely, perception of geometric figures used as controls remained overall stable in fasting as compared to satiety. Therefore, hunger is able to specifically produce modifications in the perception of food pictures, an effect presumably related to changes in their incentive effects. This is suggested by studies showing that visual and time perceptions are both modulated by the affective state in response to the presentation of food or word-related stimuli (Ode et al. 2012; Gil et al. 2009).

Our data illustrate the interaction between the physiological hunger and motivation. Prolonged fasting is associated with an increased activity within the hypothalamus (Tataranni et al. 1999) but also evokes midbrain activation in response to the anticipated experience of a forthcoming meal (DelParigi et al. 2005). Such activation is assumed to mediate the motivational aspects related to the expectation of food (Berridge 1996; Berridge 2003; Finlayson et al. 2007; Finlayson and Dalton 2012; Salamone and Correa 2002; Salamone and Correa 2012). The motivation is characterized by the assignment of attractive and desirable properties to an external stimulus such as a food image and it is mediated by the release of dopamine within the mesolimbic pathways (Berridge 1996; Berridge 2003; Finlayson et al. 2007; Finlayson and Dalton 2012; Salamone and Correa 2002; Salamone and Correa 2012). Interestingly, the mesencephalic dopamine system has also been described to occupy a pivotal position in the perception of time, according to the classical pacemakeraccumulator model that allows the estimation, integration and discrimination of time intervals (Buhusi and Meck 2005; Meck et al. 2008).

Our global evaluation of the appetitive value of the food images used in our study accounted for their incentive value. However, numerous functional neuroimaging studies have shown that the presentation of visual food stimuli is associated with the activation of frontal-limbic loops (Stoeckel et al. 2008; Stoeckel et al. 2009) that are highly involved in processing the hedonic significance of environmental stimuli (Krawczyk 2002; Phillips et al. 2003). Visual food stimuli do not only induce incentive, but also affective responses, which reflect the pleasantness 
of the sensation produced by the presentation of food images (Cabanac 1971; Brondel and Cabanac 2007). Also, our data confirm previous findings showing that the time of presentation of food images is underestimated, as compared to that of neutral pictures. Importantly, this effect is related to the pleasure provoked by the food images (Gil et al. 2009). Thus, although emotional responses were not specifically assessed in our study, it cannot be ruled out that hunger will affect not only the incentive, but also the hedonic characteristics of the food pictures.

The present study has some limitations. First, differently from what initially expected, the geometric figures used in our study, were overestimated in size as the food images, especially under fasting, when compared to their respective devalued counterparts. This finding is consistent with earlier studies showing that the color of a stimulus affects its size perception (Tedford et al. 1977; Ling and Hurlbert 2004). Relationships between the color and the emotional reaction elicited by the presentation of a stimulus have also been established (Valdez and Mehrabian 1994). Therefore, it can be assumed that the geometric figures in color relative to their devalued counterparts in grayscale could possibly acquire emotional salience, as seen for the food images, and consequently induce changes in size perception under fasting, as suggested by the effects of affective states on size estimation (Ode et al. 2012). Second, measurements of the PSD showed that hunger specifically induces an overestimation of the size of the viewed food images under fasting while assessments of PSE revealed similar changes in the size perception although occurring indifferently for both types of stimuli in fasting. This partial discrepancy between the PSD and PSE could be possibly due to the chosen psychophysical parameters, especially the size of the step, which might be too large for identifying with the PSE small perceptual differences between food and control stimuli in a sample of healthy subjects free from any pathology of the reward system. Thus, it might be necessary to further reduce the size of the step in order to better differentiate changes in the size perception of the food pictures from those of control images by using the PSE. An alternative explanation is the smaller number of subjects performing the task A than those participating in the task B for which the PSE and the PSD concordantly showed that under fasting the time of presentation was perceived shorter for food but not for control images. Third, the PSD is a particularly appropriate measurement derived from the responses to a large number of trials (from 200 to 350) where both stimuli "F" and "D" are equal in terms of size and duration of presentation, as those responses obtained in our study for each experimental session at the level of the entire group sample. However, this experimental variable might partially loose its accuracy when calculated individually for each participant on a smaller number of trials (from 9 to 14) during each experimental session, thereby resulting in the absence of correlation with the hunger levels or the appetitive value of the food images. Fourth, as reported above, the appetitive value of the viewed food images was rated on VAS at the end of the last experimental session in order to avoid giving the participants particular information about the exact objectives of the study, and therefore limiting biased responses to food images. However, this approach raises the question about the accuracy of a retrospective measurement of the overall appetitive properties attributed to the food pictures of the study. This might explain why i) there was no influence of fasting on the appetitive value of food images; and, ii) there was no relation with the experimental variables PSE and PSD that we used. Fifth, previous findings have shown the allocation of attentional resources in response to the salience and relevance of food-related stimuli (Di Pellegrino et al. 2011; Forestell et al. 2012; Yokum et al. 2011). In particular, it has been demonstrated that the attentional processing for food stimuli is influenced by fasting (Nijs et al. 2010; Siep et al. 2009; Piech et al. 2010). Our study assessed the accuracy of perception, as reflected by the PSE. However, we did not assess the precision of perception, which reflects, at least in part, the participant's attentional engagement. Finally, our sample was characterized by an overrepresentation of women. This could impact our findings, as attested by differential effects of the hunger drive on hedonic responses to food pictures according to gender (Stoeckel et al. 2007). Interestingly, such gender effect seems to depend upon the categories of food (Stoeckel et al. 2007). Thus, the use of a large variety of food stimuli as done in our study might have minimized the effects of gender. However, it might be important to examine the putative presence of gender effects in future studies requiring larger samples of subjects.

\section{Conclusion}

The present pilot study pleads for the potential usefulness of a novel computer-based test that we have developed for the study of motivational processing of food images, allowing assessing changes in visual and time perception in humans. We showed that the accuracy of the perception depends on the appetitive properties of the food stimuli and that this is in close relation with the hunger drive. This computer-based test could therefore contribute to the characterization of disturbances in reward processes and responses to standard therapeutic strategies in subjects affected by mood, addictive disorders or obesity.

\section{Methods}

Subjects

Thirty healthy subjects (11 men, 19 women), whose ages ranged from 25 to 58 years (mean age $=32.17$ 
years \pm sem $=1.78$ ), were recruited through printed announcements and word of mouth. All the subjects were free of: i) past and current DSM-IV axis I psychiatric disorders, including drug addiction or abuse; ii) serious medical and neurological disorders; and, iii) chronic exposure to psychotropic agents and other medications affecting the physiology of the central nervous system. The Body Mass Index (BMI) calculated for each participant before entering the study was systematically comprised between 18.5 and $24.9 \mathrm{~kg} / \mathrm{m}^{2}$ (mean BMI $=21.80$ $\mathrm{kg} / \mathrm{m}^{2} \pm \mathrm{sem}=0.32$ ). Also, the subjects had normal or corrected-to-normal vision. They were asked to abstain from alcohol drinking for at least 2 days before and throughout the study. Caffeine or tobacco use was not permitted over the last 3 hours prior to the testing in order to substantially reduce the risk for attention and memory biases. The INSERM Institutional Review Board specifically approved the present study. All the participants gave written informed consent after a complete description of the protocol. However, the subjects were unaware of the exact nature of the study. They were financially compensated for their participation in the study.

\section{Computer-based tasks}

Two computer-generated tasks were used. They are both registered under the French agency for the protection of computer software. The tasks were based on the discrimination of the size (Task A, Figure 3A) or of the time of presentation (Task B, Figure 3B) of food ("F", food picture) or control ("C", geometric image) stimuli and their devalued counterpart ("D") in grayscale. Devalued images were used since earlier studies demonstrated that the visual characteristics, particularly in terms of colors, have an impact on the affective reaction to salient cues, thereby influencing the size and time perception (Smets 1969; Tedford et al. 1977; Ling and Hurlbert 2004; Valdez and Mehrabian 1994; Gil et al. 2009). Images were chosen within a library containing 70 photographs of various food categories (snacks, meats, fish, pizzas, sandwiches, cheeses, fruits, and cakes) and the same number of geometric shapes. At each trial, the stimuli were presented in a random order on a computer screen so that food or geometric images in color preceded or followed their devalued counterparts. The consecutive presentation of food and geometric images throughout the task were also random. Each trial started with the presentation of a single image " $F$ ", "C" or "D" for $1 \mathrm{~s}$. After a 750-ms interval, the subject had access to the subsequent image " $\mathrm{D}$ ", " $\mathrm{F}$ " or " $\mathrm{C}$ " for $1 \mathrm{~s}$. For the task $\mathbf{A}$, the subject had to compare the images by pressing within a 3-s delay the left button of the keyboard when the first image was estimated greater, in terms of size, than the second one or the right button when the second image was judged greater that the first one. For the task B, the subject had to compare the images by pressing within a 3-s delay the left button of the keyboard when the time of presentation of the first image was estimated longer than that of the second one, or the right button when the time of presentation of the second image was judged longer than that of the first one. The presentation time of the images ranged from 500 to $1500 \mathrm{~ms}$ throughout the task.

The adopted experimental paradigm used to develop the computer-based program was derived from the classical psychophysical up-down adaptive staircase method (Jesteadt 1980). Two staircases, respectively called $\alpha$ and $\beta$, were interleaved throughout the task in an alternative manner (Figure 4). The ratio "F"/“ $\mathrm{D}$ " or " $\mathrm{C}$ "/ $\mathrm{D}$ " at each trial depended on the subject's response on the previous trial. If the subject perceived that the size or the presentation time of the stimulus " $\mathrm{F}$ " or "C" was respectively greater or longer than that of the devalued counterpart "D", the ratio " $\mathrm{F}$ "/ $\mathrm{D}$ " or " $\mathrm{C}$ "/ $\mathrm{D}$ " was reduced by one step at the subsequent trial. Conversely, it was increased by one step when the size or the presentation time of the stimulus " $\mathrm{F}$ " or " $\mathrm{C}$ " was considered smaller or shorter than that of the devalued counterpart " $\mathrm{D}$ ". The procedure is terminated after a specified number of reversals $(n=12)$ within each staircase $\alpha$ and $\beta$ (Figure 4).

\section{Experimental sessions}

Two experimental sessions in satiety were carried out 3-4 days apart in order to ensure that the behavioral responses to the tasks remained stable over time. One additional session in fasting for 6 hours before the test was performed 3-4 days apart from those in satiety. The experimental sessions in satiety preceded or followed the one in fasting in a randomized order. When tested in satiety, the subjects consumed a calorically-defined meal (600 kcal) 15 minutes after the arrival at the laboratory. Following a one-hour period of resting, they were asked to perform tasks A and B with a 20-min time interval between tasks. The order of the tasks A and B was randomized across the experimental sessions. Before starting the first task, a visual analogue scale (VAS) was systematically completed in order to assess hunger levels. Each subject had to answer the following question: "How hungry are you?" and rate hunger levels by placing a mark on a horizontal line, $100 \mathrm{~mm}$ in length, anchored by the word descriptors "Not at all" on the left end (0) and "Extremely" on the right end (100). An additional VAS evaluating the appetitive properties of the viewed food pictures was administered although only at the end of the last experimental session, so that the exact objective of the study remained unknown to the participants, thereby limiting potential response bias. The following question was asked: "How much did you consider the presented food 

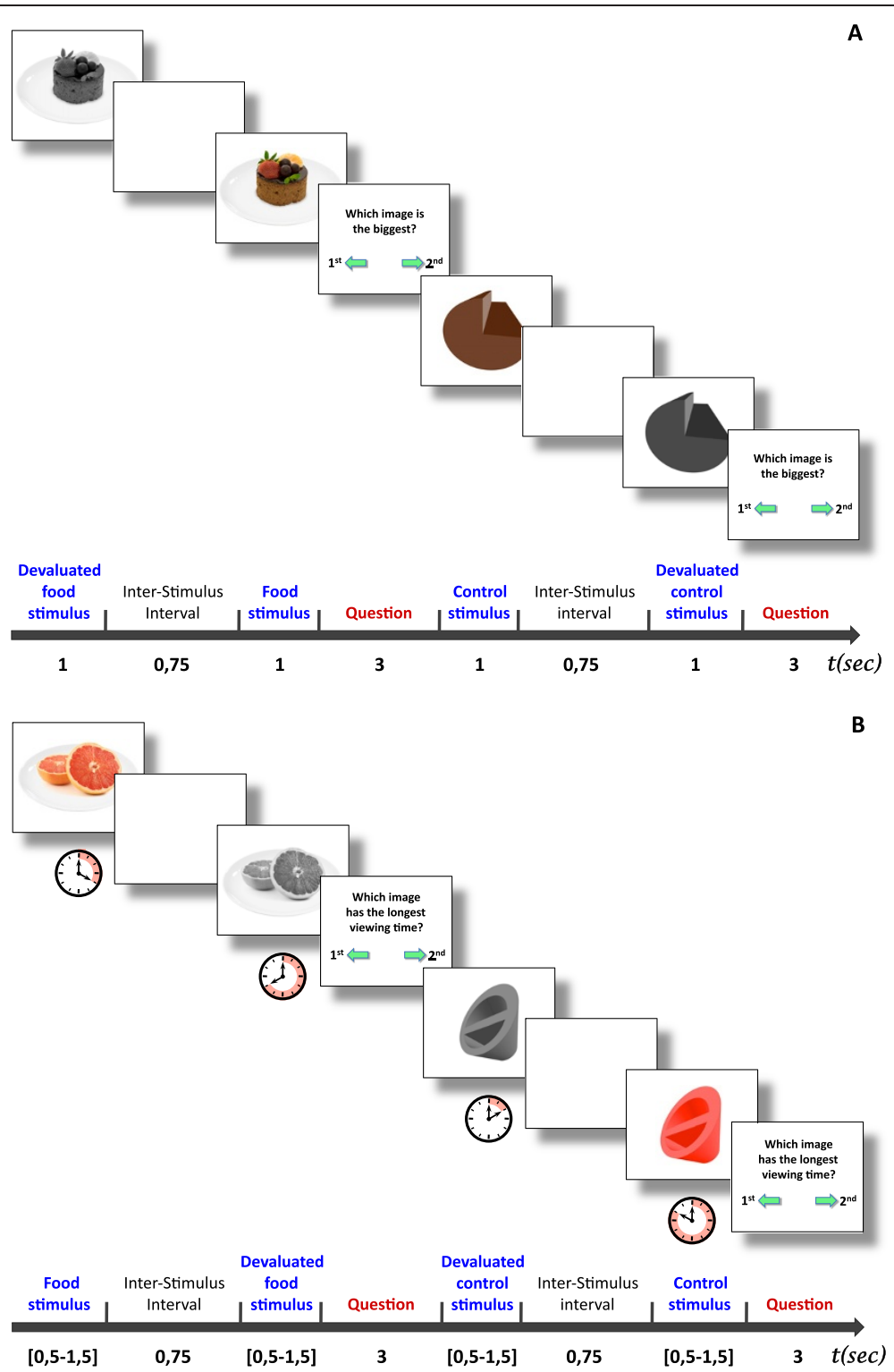

Figure 3 Illustration of tasks $\mathbf{A}$ and $\mathbf{B}$, which assess respectively visual and time discrimination capacities. (A) For task $A$, at each trial, the subject was asked to compare the size of the food stimulus (food picture in color) to that of its devalued counterpart (same image in grayscale), and to answer the following question: "Which image is the biggest?" by pressing the left or the right button (arrow key of the standard computer keyboard) when respectively the first or the second image was considered as the biggest one. Geometric figures (in a range of colors close to that of food images) and their respective devalued counterparts (in grayscale) were used as controls. For further details see Methods. (B) For task B, at each trial, the subject was asked to compare the duration of presentation of the food stimulus (food picture in color) to that of its devalued counterpart (same image in grayscale), and to answer the following question: "Which image has the longest viewing time?" by pressing the left or the right button (arrow key of the standard computer keyboard) when respectively the first or the second image was estimated as having the longest time of presentation. Geometric figures (in a range of colors close to that of food images) and their respective devalued counterparts (in grayscale) were used as controls. For further details see Methods.

images appetitive?". The subject was invited to place a mark on the horizontal line anchored by word descriptors similar to those cited above for the VAS assessing hunger levels. For both VAS, the score was determined by measuring the distance in millimeters from the left end of the line to the mark that the subject drew (Wewers and Lowe 1990; Gould et al. 2001).

\section{Data analysis}

Two experimental variables were considered as a measurement of perceptual changes that are putatively reflective of changes in motivational states. First, the point of subjective equality (PSE) calculated for each category of stimuli " $F$ " and "C" is the ratio "F"/ $D$ " or "C"/ $D$ " for which the stimulus " $\mathrm{F}$ " or " $\mathrm{C}$ " was judged equal to " $\mathrm{D}$ " in 


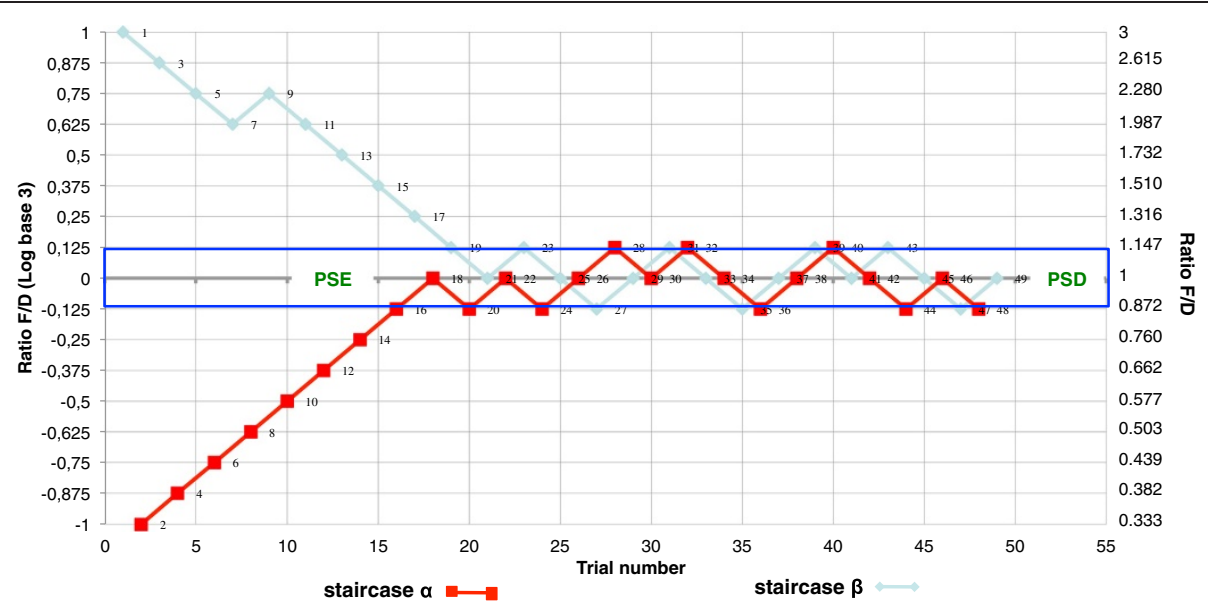

Figure 4 Illustration of the up-down adaptive staircase procedure. In this fictitious block of trials, the subject had to compare, on each trial, two stimuli, "F" and " $D$ ". The ratio F/D varied from trial to trial and was represented in two interleaved adaptive staircases called a and $\beta$. For task A (size comparisons), F/D was initially equal to 2 for staircase $a$ and $1 / 2$ for staircase $\beta$. In both staircases, F/D was subsequently multiplied by $2^{1 / 12}$ (i.e., approximately 1.059) when subjects perceived $F$ as smaller than $D$, and divided by the same factor when subjects perceived $F$ as larger than $D$. The block of trials was terminated when at least 12 reversals in the variation of F/D had occurred for each staircase. Similarly, for task B (duration comparisons), as shown in the figure, the initial values of F/D were always 3 and 1/3, and F/D was always multiplied or divided by a factor of $3^{1 / 8}$ (i.e., approximately 1.147 ) when $\mathrm{F}$ was estimated respectively shorter or longer than $\mathrm{D}$.

terms of size or presentation time and it is estimated by averaging reversal points within both staircases (Jesteadt 1980). Second, the percentage of subjective discrimination (PSD) determined for each type of stimuli " $\mathrm{F}$ " and " $\mathrm{C}$ " corresponds to the percentage of responses where the stimulus " $\mathrm{F}$ " or " $\mathrm{C}$ " was judged greater than " $\mathrm{D}$ " during the trials where the stimulus " $\mathrm{F}$ " or " $\mathrm{C}$ " was physically equal to " $\mathrm{D}$ ", in terms of size or duration of presentation (Figure 4). This latter variable is expected to show changes in either size or time perception similar to those found with the PSE. For instance, if the PSE for the stimuli "F" has a positive value, it means that the stimuli " $\mathrm{F}$ " are considered equal to their devalued counterparts " $D$ " whereas the stimuli " $F$ " are in reality greater than " $D$ " in either size or presentation time. Therefore, the stimuli " $F$ " were underestimated as compared to " $\mathrm{D}$ ". Consequently, the corresponding PSD will have a value below $50 \%$.

Of the study population, data of the first 6 subjects enrolled for task A that served for gradual psychophysical parameter adjustments (step size, initial ratio "F"/“D” or "C"/“D”) were excluded from the final analyses. For task B, data of 3 subjects were excluded because pictures were abnormally displayed on the computer screen when these 3 subjects passed the test.

One- and two-way ANOVAs were respectively performed for the comparison of: i) the hunger levels and appetitive values of food images between fasting and satiety conditions; and, ii) the PSE and PSD between fasting and satiety conditions according to the category of stimuli "F" and "C". Newman-Keuls test was used for post-hoc analysis. Pearson's test was used for correlation analyses between: 1) the hunger levels and the PSE or PSD calculated for either the stimuli " $\mathrm{F}$ " or " $\mathrm{C}$ " in the fasting condition; and, 2) the appetitive value of the stimuli " $F$ " and the PSE or PSD calculated for the stimuli "F" in the fasting condition. Results were considered as significant at $\mathrm{p}<0.05$.

\section{Abbreviations}

PSE: Point of subjective equality; PSD: Percentage of subjective discrimination; VAS: Visual analogue scale.

\section{Competing interests}

The authors declare that they have no competing interests.

\section{Authors' contributions}

$B A, C G, L D, P V P$ and DC wrote the manuscript. BA, CS, LD, PVP and DC contributed to the development of the computer-based tasks. BA, CG, LD, PVP and DC analyzed the data. CG, OD and PP participated in the recruitment and assessment of the study subjects. All the authors reviewed and approved the publication.

\section{Authors' information}

Pier Vincenzo Piazza and Daniela Cota share senior authorship.

\section{Acknowledgements}

This study was supported by INSERM (D.C., P.V.P.), Fondation pour la Recherche Médicale Master fellowship (C.G.), Servier/Eutherapie laboratories, French "Fonds Français pour I'Alimentation et la Santé (B.A.) and Labex BRAIN ANR-10-LABX-43 (D.C.). All the funders had no further role in the study design, data collection and analysis, decision to publish, or preparation of the manuscript. We thank Mrs. Bert-Latrille from the GENPPHASS for helping with the pre-screening of the potential research participants. We thank the subjects who have participated in the study.

\section{Author details}

${ }^{1}$ Regional medical center for the management and treatment of anxiety and depressive disorders, Centre Hospitalier Charles Perrens, F-33076 Bordeaux, France. ${ }^{2}$ INSERM, Neurocentre Magendie, Physiopathologie de la Plasticité Neuronale, U862, F-33000 Bordeaux, France. ${ }^{3}$ Université de Bordeaux, F-33000 
Bordeaux, France. ${ }^{4}$ Study group "Neurophysiology, pharmacology, sleep and sleepiness", CHU de Bordeaux, F-33076 Bordeaux, France. ${ }^{5}$ Group "Auditory perception and development", CNRS UMR 5287, Institut de Neurosciences Cognitives et Intégratives d'Aquitaine, F-33076 Bordeaux, France. ${ }^{6}$ Group "Energy Balance and Obesity", INSERM U862, Neurocentre Magendie, 146 Rue Léo Saignat, F-33077 Bordeaux, France.

\section{Received: 10 February 2014 Accepted: 18 July 2014}

Published: 11 August 2014

\section{References}

Berridge, KC. (1996). Food reward: brain substrates of wanting and liking. Neuroscience and Biobehavioral Reviews, 20(1), 1-25.

Berridge, KC. (2003). Pleasures of the brain. Brain and Cognition, 52(1), 106-128.

Born, JM, Lemmens, SG, Martens, MJ, Formisano, E, Goebel, R, \& WesterterpPlantenga, MS. (2011). Differences between liking and wanting signals in the human brain and relations with cognitive dietary restraint and body mass index. American Journal of Clinical Nutrition, 94(2), 392-403.

Brondel, L, \& Cabanac, M. (2007). Alliesthesia in visual and auditory sensations from environmental signals. Physiology and Behavior, 91(2-3), 196-201.

Bschor, T, Ising, M, Bauer, M, Lewitzka, U, Skerstupeit, M, Muller-Oerlinghausen, B, \& Baethge, C. (2004). Time experience and time judgment in major depression, mania and healthy subjects. A controlled study of 93 subjects. Acta Psychiatrica Scandinavica, 109(3), 222-229.

Buhusi, CV, \& Meck, WH. (2005). What makes us tick? Functional and neural mechanisms of interval timing. Nature Review Neuroscience, 6(10), 755-765.

Cabanac, M. (1971). Physiological role of pleasure. Science, 173(4002), 1103-1107.

Cooke, LJ, Chambers, LC, Anez, EV, Croker, HA, Boniface, D, Yeomans, MR, \& Wardle, J. (2011). Eating for pleasure or profit: the effect of incentives on children's enjoyment of vegetables. Psychological Science, 22(2), 190-196.

Cota, D, Tschop, MH, Horvath, TL, \& Levine, AS. (2006). Cannabinoids, opioids and eating behavior: the molecular face of hedonism? Brain Research Reviews, 51(1), 85-107.

DelParigi, A, Chen, K, Salbe, AD, Reiman, EM, \& Tataranni, PA. (2005). Sensory experience of food and obesity: a positron emission tomography study of the brain regions affected by tasting a liquid meal after a prolonged fast. Neurolmage, 24(2), 436-443.

Di Pellegrino, G, Magarelli, S, \& Mengarelli, F. (2011). Food pleasantness affects visual selective attention. Quarterly Journal of Experimental Psychology, 64(3), 560-571

Eaton, WW, Kalaydjian, A, Scharfstein, DO, Mezuk, B, \& Ding, Y. (2007). Prevalence and incidence of depressive disorder: the Baltimore ECA follow-up, 1981-2004. Acta Psychiatrica Scandinavica, 116(3), 182-188.

Epstein, LH, Saad, FG, Handley, EA, Roemmich, JN, Hawk, LW, \& McSweeney, FK. (2003). Habituation of salivation and motivated responding for food in children. Appetite, 41(3), 283-289.

Finlayson, G, \& Dalton, M. (2012). Current progress in the assessment of 'liking' vs. 'wanting' food in human appetite. Comment on "'You say it's liking, i say it's wanting...". On the difficulty of disentangling food reward in man'. Appetite, 58(1), 373-378.

Finlayson, G, King, N, \& Blundell, JE. (2007). Liking vs. wanting food: importance for human appetite control and weight regulation. Neuroscience and Biobehavioral Reviews, 31(7), 987-1002.

Forestell, CA, Lau, P, Gyurovski, II, Dickter, CL, \& Haque, SS. (2012). Attentional biases to foods: the effects of caloric content and cognitive restraint. Appetite, 59(3), 748-754.

Giesen, JC, Havermans, RC, Douven, A, Tekelenburg, M, \& Jansen, A. (2010). Will work for snack food: the association of BMl and snack reinforcement. Obesity, 18(5), 966-970.

Gil, S, Rousset, S, \& Droit-Volet, S. (2009). How liked and disliked foods affect time perception. Emotion, 9(4), 457-463.

Gould, D, Kelly, D, Goldstone, L, \& Gammon, J. (2001). Examining the validity of pressure ulcer risk assessment scales: developing and using illustrated patient simulations to collect the data. Journal of Clinical Nursing, 10(5), 697-706.

Jesteadt, W. (1980). An adaptive procedure for subjective judgments. Perception \& Psychophysics, 28(1), 85-88.

Krawczyk, DC. (2002). Contributions of the prefrontal cortex to the neural basis of human decision making. Neuroscience and Biobehavioral Reviews, $26(6), 631-664$

Ling, Y, \& Hurlbert, A. (2004). Color and size interactions in a real 3D object similarity task. Journal of Visualization, 4(9), 721-734.
Meck, WH, Penney, TB, \& Pouthas, V. (2008). Cortico-striatal representation of time in animals and humans. Current Opinion in Neurobiology, 18(2), 145-152.

Merikangas, KR, \& McClair, VL. (2012). Epidemiology of substance use disorders. Human Genetics, 131(6), 779-789.

Mezey, AG, \& Cohen, SI. (1961). The effect of depressive illness on time judgment and time experience. Journal of Neurology, Neurosurgery, and Psychiatry, 24, 269-270.

Nijs, IM, Muris, P, Euser, AS, \& Franken, IH. (2010). Differences in attention to food and food intake between overweight/obese and normal-weight females under conditions of hunger and satiety. Appetite, 54(2), 243-254.

Ode, S, Winters, PL, \& Robinson, MD. (2012). Approach motivation as incentive salience: perceptual sources of evidence in relation to positive word primes Emotion, 12(1), 91-101.

Phillips, ML, Drevets, WC, Rauch, SL, \& Lane, R. (2003). Neurobiology of emotion perception l: the neural basis of normal emotion perception. Biological Psychiatry, 54(5), 504-514.

Piazza, PV, Lafontan, M, \& Girard, J. (2007). Integrated physiology and pathophysiology of CB1-mediated effects of the endocannabinoid system. Diabetes \& Metabolism, 33(2), 97-107.

Piech, RM, Pastorino, MT, \& Zald, DH. (2010). All I saw was the cake. Hunger effects on attentional capture by visual food cues. Appetite, 54(3), 579-582.

Salamone, JD, \& Correa, M. (2002). Motivational views of reinforcement: implications for understanding the behavioral functions of nucleus accumbens dopamine. Behavioural Brain Research, 137(1-2), 3-25.

Salamone, JD, \& Correa, M. (2012). The mysterious motivational functions of mesolimbic dopamine. Neuron, 76(3), 470-485.

Siep, N, Roefs, A, Roebroeck, A, Havermans, R, Bonte, ML, \& Jansen, A. (2009). Hunger is the best spice: an fMRI study of the effects of attention, hunger and calorie content on food reward processing in the amygdala and orbitofrontal cortex. Behavioural Brain Research, 198(1), 149-158.

Smets, G. (1969). Time expression of red and blue. Perceptual and Motor Skills, 29(2), 511-514.

Stoeckel, LE, Cox, JE, Cook, EW, 3rd, \& Weller, RE. (2007). Motivational state modulates the hedonic value of food images differently in men and women. Appetite, 48(2), 139-144.

Stoeckel, LE, Weller, RE, Cook, EW, 3rd, Twieg, DB, Knowlton, RC, \& Cox, JE. (2008). Widespread reward-system activation in obese women in response to pictures of high-calorie foods. Neurolmage, 41(2), 636-647.

Stoeckel, LE, Kim, J, Weller, RE, Cox, JE, Cook, EW, 3rd, \& Horwitz, B. (2009). Effective connectivity of a reward network in obese women. Brain Research Bulletin, 79(6), 388-395.

Tataranni, PA, Gautier, JF, Chen, K, Uecker, A, Bandy, D, Salbe, AD, Pratley, RE, Lawson, M, Reiman, EM, \& Ravussin, E. (1999). Neuroanatomical correlates of hunger and satiation in humans using positron emission tomography. P National Academic Science USA, 96(8), 4569-4574.

Tedford, WH, Jr, Bergquist, SL, \& Flynn, WE. (1977). The size-color illusion. Journal of General Psychology, 97(1st Half), 145-149.

Valdez, P, \& Mehrabian, A. (1994). Effects of color on emotions. Journal of Experimental Psychology: General, 123(4), 394-409.

Wang, YC, McPherson, K, Marsh, T, Gortmaker, SL, \& Brown, M. (2011). Health and economic burden of the projected obesity trends in the USA and the UK. Lancet, 378(9793), 815-825.

Wewers, ME, \& Lowe, NK. (1990). A critical review of visual analogue scales in the measurement of clinical phenomena. Research in Nursing and Health, 13(4), 227-236.

Wyrick, RA, \& Wyrick, LC. (1977). Time experience during depression. Archives of General Psychiatry, 34(12), 1441-1443.

Yokum, S, Ng, J, \& Stice, E. (2011). Attentional bias to food images associated with elevated weight and future weight gain: an fMRI study. Obesity, 19(9), 1775-1783.

\section{doi:10.1186/s40359-014-0023-6}

Cite this article as: Aouizerate et al:: Toward a new computer-based and easy-to-use tool for the objective measurement of motivational states in humans: a pilot study. BMC Psychology 2014 2:23. 